\title{
The role of L-carnitine in nutritional status and echocardiographic parameters in idiopathic dilated cardiomyopathy in children
}

\author{
Vitor M. P. Azevedo, ${ }^{1}$ Francisco M. Albanesi Filho, ${ }^{2}$ Marco A. Santos, ${ }^{3}$ \\ Márcia B. Castier, ${ }^{4}$ Maria Ourinda M. Cunha ${ }^{5}$
}

\begin{abstract}
Objectives: Malnutrition is an independent predictor of death in idiopathic dilated cardiomyopathy. An analysis was performed of the impact of L-carnitine supplementation on the nutritional status and echocardiogram parameters of children with idiopathic dilated cardiomyopathy.

Methods: This was an open label cohort of 11 patients who received L-carnitine $(100 \mathrm{mg} / \mathrm{kg} / \mathrm{day})$ plus the conventional medical treatment, compared with 40 controls, matched for gender and age. The L-carnitine group was weighed 118 times and the controls 264 times. Additionally, the L-carnitine group underwent 65 two-dimensional echocardiograms and the controls 144. Chi-square, Student's t test, Pearson correlation and ANOVA were calculated with alpha $=0.05$.

Results: For the L-carnitine group: age at presentation $=3.82$ years old, $72.7 \%(p=0.033)$ were females younger than 2 years and $90.9 \%(p=0.0001)$ were in functional classes III and IV. There were no deaths during this period. At presentation, no differences were observed in weight percentile $(31.2 \pm 8.74$ vs. $19.6 \pm 21.2)(p=0.29)$ or $z$ score $(-0.68 \pm 1.05$ vs. $-1.16 \pm 0.89)(p=0.24)$. Increases were observed in both the percentile $(p=0.026)$ and $z$ score $(p=0.033)$ after the introduction of $L$-carnitine. At presentation, there were no differences in ejection fraction $(54.9 \% \pm 3.8$ vs. $49.3 \% \pm 6.6)(p=0.19)$, but LV mass/BSA were greater in the L-carnitine group $\left(169.12 \mathrm{~g} / \mathrm{m}^{2} \pm 26.24\right.$ vs. $\left.110.67 \mathrm{~g} / \mathrm{m}^{2} \pm 15.62\right)(\mathrm{p}=0.0005)$. After the introduction of L-carnitine an increase in ejection fraction $(48.3 \pm 7$ to $67.2 \pm 7)(p=0.044)$ was observed. LV mass/BSA decreased $\left(164.29 \mathrm{~g} / \mathrm{m}^{2} \pm 28.14\right.$ to $\left.110.88 \mathrm{~g} / \mathrm{m}^{2} \pm 28.88\right)$, but without significance $(p=0.089)$
\end{abstract}

Conclusion: In children with idiopathic dilated cardiomyopathy, supplementation of L-carnitine may be helpful for nutritional and echocardiographic improvement.

J Pediatr (Rio J). 2005;81(5):368-72: Acetylcarnitine, congestive cardiomyopathy, child, malnutrition, congestive heart failure, echocardiography.

\section{Introduction}

Heart failure (HF) can be defined as a clinical syndrome in which the heart is unable to adequately fulfill its pumping function and cannot supply enough oxygenated blood to

1. MSc. PhD student, Universidade do Estado do Rio de Janeiro (UERJ). Pediatric cardiologist, Instituto Nacional de Cardiologia Laranjeiras, Rio de Janeiro, RJ, Brazil.

2. Professor, UERJ, Rio de Janeiro, RJ, Brazil.

3. MSc. Pediatric cardiologist, Instituto Nacional de Cardiologia Laranjeiras, Rio de Janeiro, RJ, Brazil.

4. PhD. Professor, UERJ, Rio de Janeiro, RJ, Brazil.

5. Specialist, Sociedade Brasileira de Pediatria. Pediatrician, Intensive Care Service, National Cancer Institute, Rio de Janeiro, RJ, Brazil.

Manuscript received Jan 28 2005, accepted for publication May 252005.

Suggested citation: Azevedo VM, Albanesi Filho FM, Santos MA, Castier MB, Cunha MO. The role of L-carnitine in nutritional status and echocardiographic parameters in idiopathic dilated cardiomyopathy in children. J Pediatr (Rio J). 2005;81:368-72. meet demand necessary for normal tissue metabolism, including that necessary for growth and development. ${ }^{1}$ Standing out among the principal etiologies of childhood heart failure are congenital heart diseases, rheumatic disease, arrhythmia, myocarditis and idiopathic dilated cardiomyopathy (IDCM). ${ }^{2}$

According to the World Health Organization, dilated cardiomyopathy is characterized by dilation and inadequate contraction of the left ventricle or of both ventricles. It can be idiopathic, familial or genetic, associated or not with an innate error of metabolism, viral and/or immunological, alcoholic/toxic or associated with recognized cardiovascular diseases in which the degree of myocardial dysfunction cannot be explained by the abnormal load conditions or by ischemic damage. ${ }^{3}$ Histology is non-specific. Presentation is generally by heart failure, which is normally progressive. Arrhythmia, 
thromboembolism and sudden death are common and may occur at any stage of the disease. ${ }^{4-6}$

In the pediatric population IDCM is responsible for significant numbers of consultations and hospital admissions for heart failure unrelated to congenital heart disease and it is responsible for up to $29 \%$ of consultations below 2 years of age. ${ }^{7}$ The mortality rate is high with levels reported in the literature varying from $16 \%{ }^{8}$ over 10 years, to elevated rates such as $49 \%,{ }^{9} 66 \% 10$ and even $80 \%,{ }^{11}$ over 5 years.

Malnutrition is a severe complication of chronic diseases including cancer, the acquired immunodeficiency syndrome and chronic heart failure. For a very long time, since the era of Hippocrates (460-370 AC), 12 it has been known that weight loss, weakness and low endurance of exercise, accompanied by muscle atrophy, are part of the presentation of heart failure. We recently demonstrated that malnutrition is an independent marker for death in children and adolescents with IDCM. ${ }^{13}$

L-carnitine is a quaternary amine compound, which facilitates the transport of long chain fatty acids into mitochondria. Within the mitochondria the fatty acids enter into the beta-oxidation cycle and are converted into acetyl coenzyme A for later entry into the Krebs cycle and into the respiratory chain producing energy. ${ }^{14}$ In dilated cardiomyopathy associated with metabolic errors, such as defects of beta-oxidation and the mitochondrial disorders, there is an accumulation of intermediate organic acids. L-carnitine conjugates with these acids, removing them from the mitochondria and eliminating them in urine. Lcarnitine supplementation is the keystone of treatment for dilated cardiomyopathy secondary to beta-oxidation defects. ${ }^{15}$

Considering the above, it is presumable that L-carnitine replacement as a supplemental therapy in the treatment of patients with IDCM that is not associated with beta-oxidation disorders, could improve the energetic/metabolic performance of the remaining cardiomyocytes and the skeletal muscle cells. Such an effect could delay or even revert the process of cachexia that is so common among severe heart failure patients.

The objective of this study was to analyze the impact on nutritional parameters of introducing L-carnitine as a supplemental therapy to the treatment of children and adolescents with IDCM, in addition to analyzing the effects of L-carnitine on the ejection fraction of the left ventricle (LVEF) and on left ventricle mass/body surface area, evaluated by 2D echocardiogram.

\section{Patients and methods}

This is an open label prospective study involving 51 children (1999 to 2004) with IDCM, undertaken at the Instituto Nacional de Cardiologia in Laranjeiras. Inclusion criterion was heart failure, associated with cardiomegaly on chest $\mathrm{x}$-ray and/or dilatation of left ventricle with reduced contractility seen on 2D echocardiogram. Patients were excluded if they had congenital heart disease, anomalous origin of the coronary arteries, familial cardiomyopathy, innate errors of metabolism, Kawasaki disease, arrhythmogenic ventricular cardiomyopathy, ischemic lesion secondary to asphyxia neonatal or post cardiorespiratory resuscitation, antineoplastic drug use, primary arrhythmia, congenital valvular defects or those caused by rheumatic disease, neuromuscular disease, arterial hypertension, septicemia, HIV infection, Chagas disease or diphtheria.

Eleven of the 51 patients were randomly selected to receive $L$-carnitine and were compared with the 40 patients who did not receive medication, paired for sex, age and functional class (NYHA) at presentation. Oral L-carnitine was given at a dosage of $100 \mathrm{mg} / \mathrm{kg} /$ day in addition to the standard treatment for heart failure (digoxin, furosemide, spirinolactone, captopril and aspirin). Patients were followedup by the Nutrition Service and received nutritional guidance at the start of treatment and every 3 months, adjusting nutrition to age. The L-carnitine group was weighed 118 times during the 39 months follow-up and the control group 264 times. Calculations were made using EPINUT 2.0 - the anthropometry module of Epi-Info 6.04c by the CDC (Centers for Disease Control \& Prevention) - for weight percentile and standard deviation (SD) (z-score) for age and sex of the patients. The diagnostic criterion for malnutrition was either weight below the second SD (or $z<-2$ ) or below the fifth percentile. In addition to the weighings, serial echocardiograms were taken during follow-up. Measurements were taken of LVEF and left ventricle mass/ body surface area (LVM/BSA). Two hundred and nine echocardiograms were taken during the observation period, 65 of the L-carnitine group and 144 of the controls.

Statistical analysis was performed using Epi-Info 6.04 by the CDC and Statistica 6.0 by Statsoft Inc. Dichotomous values were evaluated with $\mathrm{c}^{2}$ (chi-square) and when applicable $95 \%$ confidence intervals (CI95) were calculated. Descriptive data was expressed as mean \pm SD and value ranges, and were analyzed with Student's $t$ test. Continuous time-dependent variables were evaluated by analysis of variance (ANOVA) for unbalanced repeated measures, by group (L-carnitine or control) and by time since clinical onset. Analysis of single continuous variables over time was performed by one-way ANOVA. Correlation between continuous variables was measured by the Pearson method. Alpha was set at 0.05 and beta at 0.80 .

Ethical Considerations: Authorization to undertake the study was obtained from the Commission for Ethics in Research at the Instituto Nacional de Cardiologia in Laranjeiras and at the Universidade do Estado do Rio de Janeiro.

\section{Results}

The average age of the L-carnitine group was 3.82 years $(0.3$ a 15.4), with $72.7 \%($ CI95 $=39.3-92.7 \%)(p=0.033)$ being under 2 years old and female and $45.4 \%$ (CI95 = $18.1-75.4 \%)(p=0.67)$ being white. The majority of the patients $(90.9 \%$; CI95 $=57.1-99.5 \%)$ were severe cases, functional classes III and IV $(p=0.001)$. There were no 
deaths during the period. There was no difference (controls vs. L-carnitine) in initial weight percentile (31.2 \pm 8.74 vs. $19.6 \pm 21.2)(p=0.29)$ or $z$-score $(-0.68 \pm 1.05$ vs. $-1.16 \pm 0.89)(p=0.24)$. The mean period from clinical onset to starting on L-carnitine was $8.6 \pm 5.7$ months. After L-carnitine was introduced, the ANOVA demonstrated a progressive increase in weight percentile (38.7 \pm 10.9 to $73.9 \pm 21.2)$ and $z$-score $(-0.23 \pm 0.46$ to $0.68 \pm 0.80)$, which was statistically significant as compared with the controls: weight percentile $(p=0.026)$ and $z$-score for weight $(p=0.033)$ (Figures 1 and 2$)$. In the control group, however, one-way ANOVA did not demonstrate any significance for weight percentile $(31.2 \pm 8.9$ to $35.3 \pm 25.0)(p=0.54)$ or $z$-score $(-0.68 \pm 0.41$ to $-0.72 \pm 1.16)(p=0.52)$. The $\mathrm{L}$-carnitine group had positive correlations for both weight percentile $(r=0.43-p<0.001)$ and $z$-score $(r=0.44-$ $p<0.001$ ) regarding time of follow-up, however in the control group no correlation was observed for the percentile $(r=0.09-p=0.25)$ or the $z$-score $(r=0.11-p=0.15)$.

At presentation, echocardiograms did not demonstrate significant differences (controls vs. L-carnitine) for LVEF $(54.9 \% \pm 3.8$ vs. $49.3 \% \pm 6.6)(p=0.19)$, but the left ventricle mass/body surface area (LVM/BSA) ratio was higher in the group that would be given L-carnitine than for the controls $\left(169.12 \mathrm{~g} / \mathrm{m}^{2} \pm 26.24\right.$ vs. $110.67 \mathrm{~g} / \mathrm{m}^{2} \pm 15.62$ ) $(p=0.0005)$ revealing more pronounced cardiomegaly at presentation. After the introduction of L-carnitine, ANOVA demonstrated a progressive increase in LVEF (48.3 \pm 7 to $67.2 \pm 7)(p=0.044)$, revealing improved cardiac performance (Figure 3 ). In the L-carnitine group LV mass reduced $\left(164.29 \mathrm{~g} / \mathrm{m}^{2} \pm 28.14\right.$ to $\left.110.88 \mathrm{~g} / \mathrm{m}^{2} \pm 28.88\right)$, but this reduction did not reach statistical significance $(p=0.089)$ when compared to the control group (Figure 4).

\section{Discussion}

Myocardial contractility primarily depends on lipid metabolism in the mitochondria for energy supply (ATP), with glucose and lactate being less important energy sources. Oxidation of fatty acids is a complex process involving 20 stages and 18 enzymes. The primary stages include the ingress of fatty acids into the mitochondria, the carnitine cycle, beta-oxidation, the Krebs cycle and ATP phosphorylation. ${ }^{15}$

L-carnitine has cardioprotective properties in the presence of hypoxia and oxidative stress in several different cardiac diseases. Its use has been beneficial to the recovery of adult patients with myocardial infarction, angina and congestive heart failure. Rizos has demonstrated an improved 3-year survival curve compared to controls in adult dilated cardiomyopathy patients who were given L-carnitine supplementation ( $2 \mathrm{~g} /$ day). ${ }^{16}$ The author states that Lcarnitine has potential in the long term treatment of dilated cardiomyopathy. In moderate to severe heart failure it increases tolerance of exercise. ${ }^{17}$

The control of heart failure with conventional medication (digoxin, furosemide, spirinolactone and captopril) allows nutrients to be better absorbed by reducing intestinal

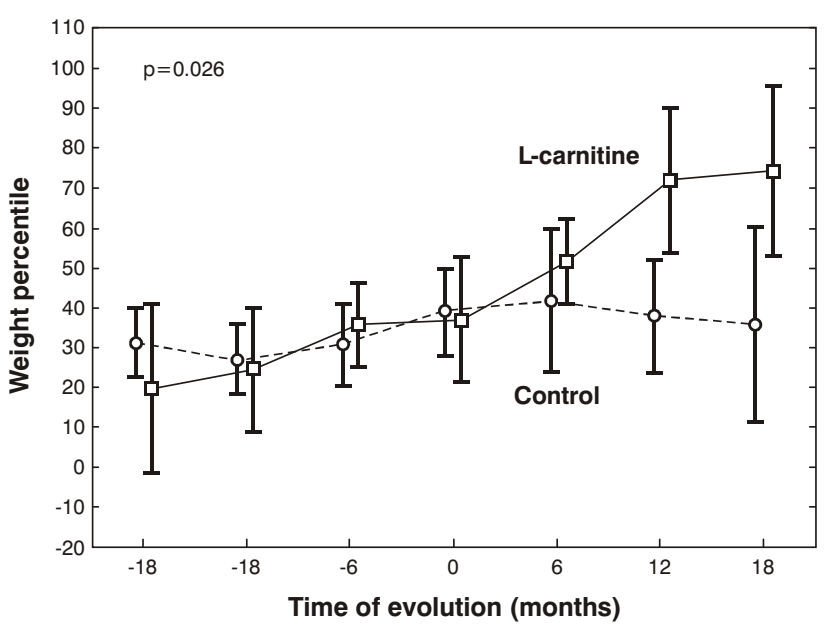

Figure 1 - Progression of the weight percentile and $95 \%$ confidence interval between groups

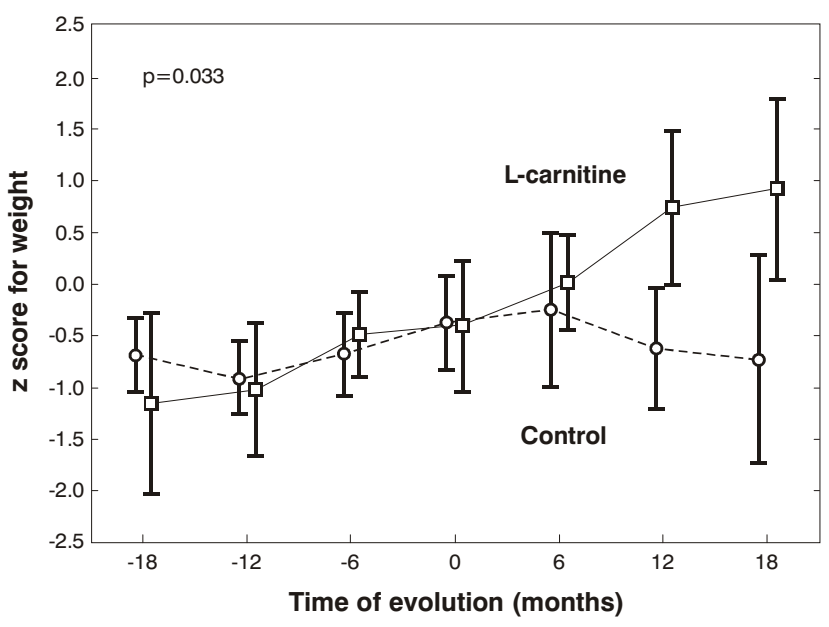

Figure 2 - Progression of the z-score for weight and $95 \%$ confidence interval between groups

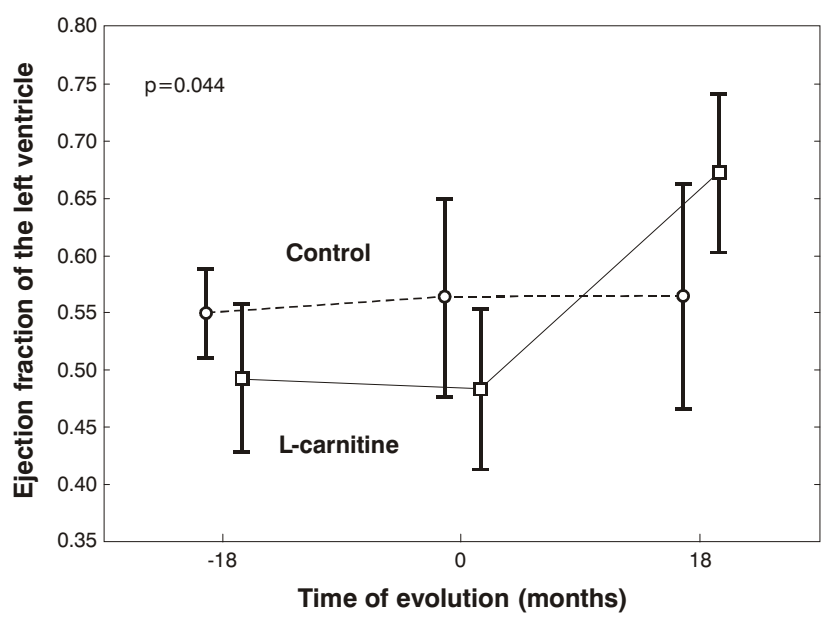

Figure 3 - Progression of the ejection fraction in the left ventricule and $95 \%$ confidence interval between groups 


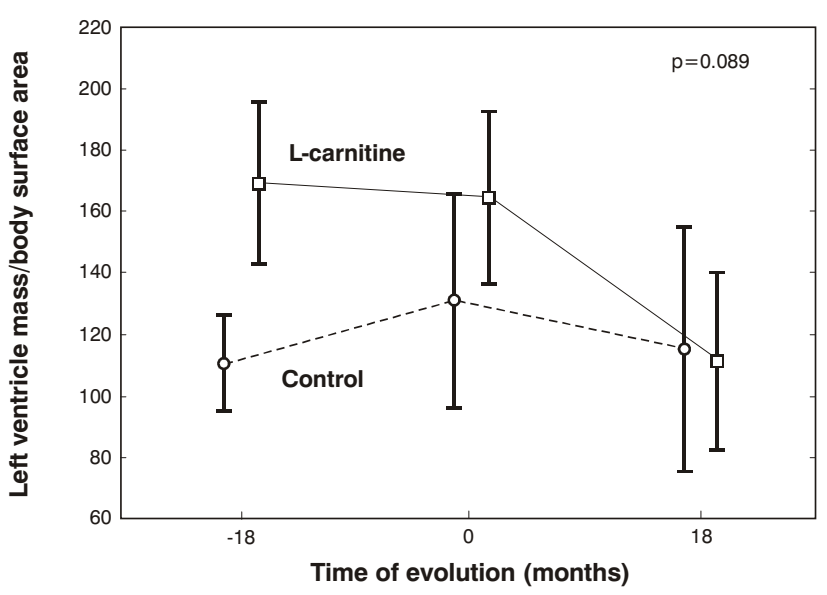

Figure 4 - Progression of the left ventricule mass/body surface area and $95 \%$ confidence interval between groups

edema, offering better energy yield and improved tissue perfusion. As a result of these factors nutritional recovery takes place with mean weight returning to close to the 50th percentile and the z-score to close to zero, as can be observed in Figures 1 and 2. Considering the beneficial effects of these medicines for nutritional recovery due to heart failure control, an average of 9 months was allowed to pass before L-carnitine was introduced. In Figures 1 and 2, the date of L-carnitine introduction is taken as the zero for abscissas. There was an additional gain in mean weight, reaching close to the 75 th percentile and taking the z-score close to 0.70 in the L-carnitine group, with no changes in cardiovascular measurements. This additional gain did not occur in the control group, suggesting that it is a beneficial effect of the L-carnitine.

The additional weight gained by the L-carnitine group could be due to extra muscle mass or just adipose tissue. Despite not having been one of the objectives of the study, upper arm and abdominal skin folds were measured and did not suggest obesity resulting from the introduction of the L-carnitine. The weight gain was primarily due to increased muscle mass which increases the capacity to exercise. ${ }^{18}$

L-carnitine has proven to be useful to echocardiographic parameters improvement with increased LVEF and a potential reduction in left ventricle mass. Since there is no regeneration of cardiomyocytes in pathophysiology of IDCM, the increased ejection fraction is probably due to better energetic/metabolic performance by the remaining cardiomyocytes with greater muscular force being generated for each contractile unit. The reduction in left ventricle mass/body surface area reflects reduced left ventricle hypertrophy, which has become unnecessary to compensate for contractile deficits. ${ }^{19}$

We should not forget that cardiomyopathy secondary to innate errors of metabolism does not only present in the dilated form, but can also occur in the familial hypertrophic form, as demonstrated by a report by Lev et al. ${ }^{20}$ However, hypertrophic cardiomyopathy progresses with an adequate nutritional status. The literature does not offer references to the use of L-carnitine for treatment of the hypertrophic form unassociated with innate errors of metabolism.

The present study suggests that L-carnitine supplementation can be of help in nutritional recovery, facilitating a reverse of the cachexia process that is common in heart failure. This supplementation should be started early, once material that diagnoses an innate error of metabolism has been collected. In certain types of innate error of metabolism, L-carnitine supplementation does not prove beneficial. ${ }^{21}$ The introduction of L-carnitine at the start of treatment for dilated cardiomyopathy in the pediatric population, after ruling out those forms of innate metabolic errors for which L-carnitine supplementation is not if benefit, has the potential to accelerate recovery of nutritional status and help to prevent or revert the cachexia that all patients with heart failure can develop.

\section{References}

1. Talner NS. Heart failure. In: Moss AJ, Adams FH. Heart disease in infants, children and adolescents. 5th ed. Baltimore: Williams \& Wilkins; 1995 . p. 1746-73.

2. O'Laughlin MP. Congestive heart failure in children. Pediatr Clin North Am. 1999;46:263-73.

3. Richardson P. Report of the 1995 World Health Organization/ International Society and Federation of Cardiology Task Force on the Definition and Classification of Cardiomyopathies. Circulation. 1996;93:841-2.

4. Günthard J. Dilated cardiomyopathy in children and thromboembolism. Eur J Pediat.r 1997;156:3-6.

5. Chang YC. Left ventricular thrombi in children with dilated cardiomyopathy. J Formos Med Assoc. 1995;94:469-73.

6. Berger S. Sudden cardiac death in infants, children and adolescents. Pediatr Clin North Am. 1999;46:221-34.

7. Matitiau A, Perez-Atayde A, Sanders SP, Sluysmans T, Parness IA, Spevak PJ, et al. Infantile dilated cardiomyopathy - relation of outcome to left ventricular mechanics, hemodynamics, and histology at the time of presentation. Circulation. 1994;90: 1310-8.

8. Friedman RA, Moak JP, Garson A. Clinical course of idiopathic dilated cardiomyopathy in children. J Am Coll Cardiol. $1991 ; 18: 152-6$.

9. Arola A, Tuominen J, Ruuskanen O, Jokinen E. Idiopathic dilated cardiomyopathy in children: prognostic indicators and outcome. Pediatrics. 1998;101:369-76.

10. Taliercio CP, Seward JB, Driscoll DJ, Fisher LD, Gersh BJ, Tajik AJ. Idiopathic dilated cardiomyopathy in the young: clinical profile and natural history. J Am Coll Cardiol. 1985;6:1126-31.

11. Akagi T, Benson LN, Lightfoot N, Chin K, Wilson G, Freedom RM. Natural history of dilated cardiomyopathy in children. Am Heart J. $1991 ; 121: 1502-6$.

12. Katz AM, Katz PB. Diseases of heart in works of Hippocrates. $\mathrm{Br}$ Heart J. 1962;24:257-64.

13. Azevedo VM, Albanesi Filho FM, Castier MB, Santos MA, Tura BR. O Impacto da desnutrição na cardiomiopatia dilatada idiopática na infância. J Pediatr (Rio J). 2004;80:211-6.

14. Pierpont ME, Breningstall GN, Stanley CA, Singh A. Familial carnitine transporter defect: a treatable cause of cardiomyopathy in children. Am Heart J. 2000;139:S96-106.

15. Helton E, Darragh R, Francis P, Fricker FJ, Jue K, Koch G, et al. Metabolic aspects of myocardial disease and a role for Lcarnitine in the treatment of childhood cardiomyopathy. Pediatrics. 2000;105:1260-70.

16. Rizos I. The role of energy metabolism defects in cardiomyopathy: from inborn errors to ischemia. Am Heart J. 2000;139:S120-3.

17. Pauly DF, Pepine CJ. The role of carnitine in myocardial dysfunction. Am J Kidney Dis. 2003;41(4 Suppl 4):S35-43.

18. Hoppel C. The role of carnitine in normal and altered fatty acid metabolism. Am J Kidney Dis. 2003;41(4 Suppl 4):S4-12. 
19. Azevedo VM, Albanesi Filho FM, Castier MB, Santos MA, Cunha MO. O impacto da L-carnitina nos parâmetros ecocardiográficos da cardiomiopatia dilatada na infância [resumo]. Rev Socerj. 2005;18 Suppl A:S100.

20. Lev D, Nissenkorn A, Leshinsky-Silver E, Sadeh M, Zeharia A, Garty BZ, et al. Clinical presentations of mitochondrial cardiomyopathies. Pediatric Cardiology. 2004;25:443-50.

21. Venditti CP, Stanley CA. Defects in metabolism of lipids. In: Behrman N. Textbook of Pediatrics. 17th ed. St. Louis: Elsevier; 2004. p. 433-7.
Correspondence:

Vitor Manuel Pereira Azevedo

Rua Visconde de Ouro Preto, 39/304, Botafogo,

CEP 22250-180 - Rio de Janeiro, RJ, Brazil

Tel.: +55212552.4123

E-mail:vitoraze@ig.com.br and vitoraze8@hotmail.com 